\title{
The Clinical Challenge of Opioid-induced Constipation: Insights from the Opioid-induced Constipation Clinical Audit
}

\author{
Peter Katelaris $^{1, *}$ and George Krassas ${ }^{2}$ \\ ${ }^{1}$ University of Sydney, Concord Clinical School, Gastroenterology and Liver Centre, Concord Hospital, Australia \\ ${ }^{2}$ Scius Healthcare Solutions Pty Ltd, Sydney, Australia
}

\begin{abstract}
Objective: Constipation is the most common and often most debilitating adverse effect associated with opioid use. Opioid-induced constipation persists for the duration of therapy. The aims of this clinical audit were to investigate the GP management of opioid-induced constipation, gain insights on how to improve its management and determine if the audit could improve the management of opioid-induced constipation.

Methods: Using quantitative questionnaires, GPs prospectively evaluated their management of constipation in patients prescribed strong opioid analgesic for chronic non-cancer pain, across two audit cycles. The audited patients completed a quantitative survey after the initial GP visit and returned the completed survey to the GP in a sealed envelope. The patient questionnaire was used to assess consistency between patients' and GPs' evaluation and management of constipation. Following each cycle GPs received feedback and a decision support tool.

Results: Opioid-induced constipation was reported by $50.5 \%$ of patients, but its presence was underestimated by GPs, with GPs failing to recognise opioid-induced constipation in a third of patients. GP management of opioidinduced constipation improved during the audit with improvements in the frequency of assessment and the proactive management of constipation.

Conclusion: GPs need to regularly ask all patients taking opioid analgesics about the presence of constipation. Questioning should include both objective and subjective measures to assist the detection and assessment of opioidinduced constipation. The high prevalence of opioid-induced constipation necessitates proactive management at the time of opioid initiation and for the duration of opioid therapy.
\end{abstract}

Keywords: Opioid-induced constipation; Constipation; Opioid; Analgesics; General practice; Clinical audit

\section{Objectives}

Constipation is the most common side effect of opioid therapy [1]. It is a consequence of opioids binding to mu-opioid receptors in the gastrointestinal tract, leading to decreased propulsive movement of bowel contents, decreased gut secretion of intestinal fluid and sphincter dysfunction [2,3]. The prevalence of opioid-induced constipation (OIC) in patients with chronic non-cancer pain (CNCP) ranges from $15 \%$ to $81 \%$ [3-7].

Unlike other opioid-related side effects, OIC does not improve over time, but persists for the duration of opioid therapy $[1,8]$. OIC is not a trivial complaint. If poorly managed it may lead to clinical sequelae, including faecal impaction with spurious diarrhoea, colonic pseudoobstruction, abdominal pain, nausea and vomiting $[9,10]$. It has a negative impact on patient quality of life and undermines effective pain management [3].

Best clinical practice recommends that OIC be proactively managed and regularly monitored $[1,11,12]$. None the less a recent survey amongst Australian GPs, found that over one third of GPs either never or only occasionally assessed opioid-related side effects, including constipation, amongst their patients with CNCP [13]. This suggests that OIC may be undiagnosed and/or inadequately managed in Australian general practice.

The aims of this clinical audit were to investigate the GP management of OIC in patients taking a strong opioid for CNCP, to gain insights on how to improve the management of this common and persistent side effect and to determine if participation in the audit will improve the management of OIC.

\section{Methods}

The OIC clinical audit followed The Royal Australian College of General Practitioners (RACGP) five-step clinical audit process: needs assessment, audit cycle 1, review and reflection, audit cycle 2 and a further review and reflection. GPs participating in this prospective audit evaluated their management of OIC in 15 patients (10 in cycle 1 and 5 in cycle 2) who were taking a strong (Schedule 8 ) opioid analgesic for $\mathrm{CNCP}$ for at least 2 weeks prior to the audit. All patients provided consent to participate in the clinical audit. Following completion of each audit cycle, GPs received feedback in the form of an individualized report on their performance versus the audit criteria and a decision support tool that summarized laxative use and categorized patients according to whether they were currently constipated or not and the impact of OIC on quality of life and pain management. A brief educational report was also provided to GPs following the completion of cycle 1 .

An educational committee comprising of six GPs and one

*Corresponding author: Peter Katelaris, Associate Professor, MBBS, FRACP MD (Lond), AGAF, Clinical Associate Professor Medicine, University of Sydney Concord Clinical School, Gastroenterology and Liver Centre, Concord Hospital, Australia, Tel: 6129767 7908; E-mail: peter.katelaris@sydney.edu.au

Received December 27, 2015; Accepted January 04, 2016; Published January 15,2016

Citation: Katelaris P, Krassas G (2016) The Clinical Challenge of Opioid-induced Constipation: Insights from the Opioid-induced Constipation Clinical Audit. J Gen Pract 4: 221. doi: 10.4172/2329-9126.1000221

Copyright: (c) 2016 Katelaris P, et al. This is an open-access article distributed under the terms of the Creative Commons Attribution License, which permits unrestricted use, distribution, and reproduction in any medium, provided the original author and source are credited. 
Citation: Katelaris P, Krassas G (2016) The Clinical Challenge of Opioid-induced Constipation: Insights from the Opioid-induced Constipation Clinical Audit. J Gen Pract 4: 221. doi: 10.4172/2329-9126.1000221

Page 2 of 5

gastroenterologist assisted with the development of the audit protocol, including setting the standards of care against which GP performance was evaluated (Table 1). The four audit criteria were developed from published reviews of the management of OIC, which represent best clinical practice [2,9-10]. GPs were recruited through the RACGP QI\&CPD website, advertisements and by personal invitation.

Information about OIC and its management was collected from both the audited patients and GPs using quantitative questionnaires. Patient assessment of constipation was assessed using stool frequency, the Bristol Stool Scale and the Bowel Function Index [2]. To ensure that the patient surveys did not influence GPs' responses, patients were instructed to complete the survey after their appointment and return the completed form to the GP in a sealed reply-paid envelope.

The audit was accredited by the RACGP and approved by the Bellberry Human Research Ethics Committee.

\section{Statistical Analysis}

All data were captured in an Access database. Only data from GPs who completed both audit cycles within the first year of the audit commencing were included in this analysis. Analysis included basic descriptive statistics and comparisons between cycle 1 and 2 results using two-tailed t-tests.

\section{Results}

A total of 64 GPs completed the audit within the first year and provided data on 956 patients (638 in cycle 1 and 318 in cycle 2). The rate of return of patient surveys was excellent at $98.7 \%(n=944)$. The characteristics of the audited patients are summarised in Table 2. Most patients were prescribed a single opioid to manage CNCP. The three most commonly prescribed opioids in cycle 1 were oxycodone $(28.2 \%)$, buprenorphine $(24.0 \%)$ and modified-release oxycodone/naloxone (16.9\%). In audit cycle 2 these same three opioids were the most commonly prescribed, however the use of modified-release oxycodone/ naloxone significantly increased and was the most commonly prescribed opioid $(24.8 \%, \mathrm{P}=0.004)$, followed by oxycodone $(24.5 \%)$ and buprenorphine (18.9\%).

The mean GP performance against the four audit criteria are shown in Figure 1. The only criteria that GPs performed well relative to the acceptable standard was criteria 3, the provision of lifestyle advice. Performance across all criteria improved significantly in cycle 2. This improvement was reflected in the percentage of individual GPs who achieved the acceptable standards across the audit criteria. For example, $35.9 \%$ of GPs informed $100 \%$ of their patients about the risk of constipation prior to initiating opioid therapy in cycle 1 , in cycle 2 this increased to $65.6 \%(\mathrm{P}=0.001)$. In cycle $1,7.8 \%$ of $\mathrm{GPs}$ co-prescribed

\begin{tabular}{|c|c|c|}
\hline Audit criteria & Ideal standard & Acceptable standard \\
\hline $\begin{array}{l}\text { 1. Patients prescribed opioid analgesics are informed of the potential side effect of constipation prior to } \\
\text { commencing opioid therapy. }\end{array}$ & $100 \%$ & $100 \%$ \\
\hline 2. At the time when opioid therapy is commenced, patients are co-prescribed a therapy to prevent/manage OIC. & $100 \%$ & $80 \%$ \\
\hline $\begin{array}{l}\text { 3. At or before opioid therapy is commenced, patients are provided with lifestyle advice to help manage } \\
\text { constipation. }\end{array}$ & $100 \%$ & $70 \%$ \\
\hline $\begin{array}{l}\text { 4. The presence and management of constipation is regularly assessed in patients prescribed opioid therapy (i.e. } \\
\text { at every appointment where the patient's pain is reviewed). }\end{array}$ & $100 \%$ & $80 \%$ \\
\hline
\end{tabular}

Table 1: OIC clinical audit evaluation criteria

Table 2: Patient characteristics. 


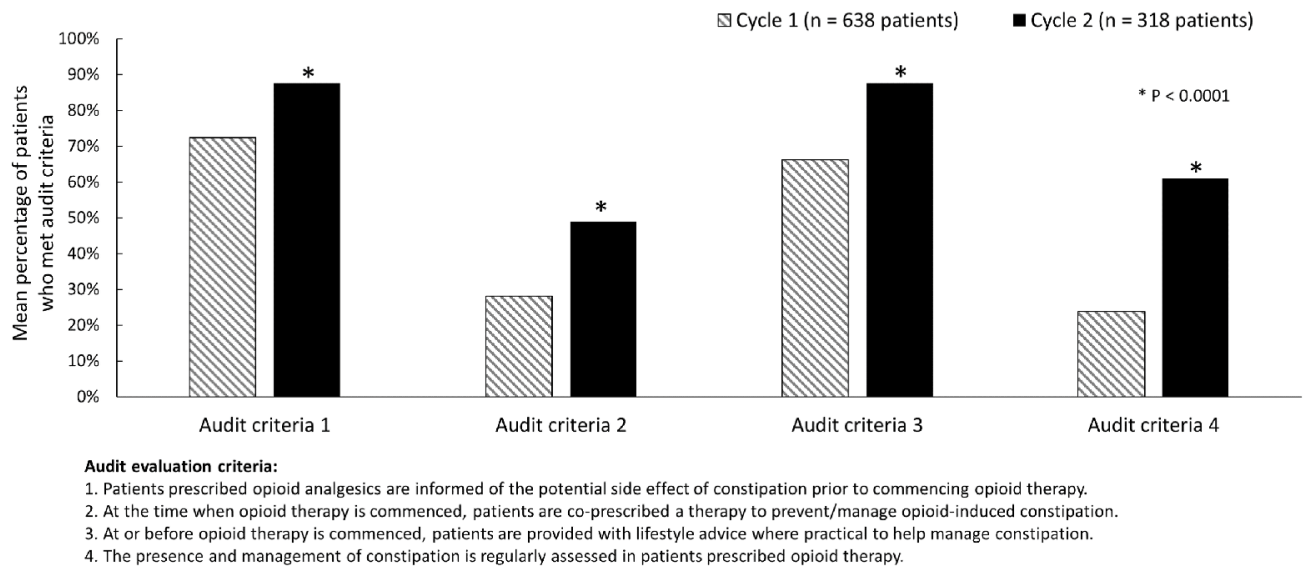

Figure 1: Mean GP performance versus audit criteria.

a therapy (opioid antagonist or laxative) to manage constipation in at least $80 \%$ of their patients at the time of opioid initiation and this increased to $26.6 \%$ in cycle $2(\mathrm{P}=0.006)$. The regular assessment of constipation in at least $80 \%$ of patients was achieved by $17.2 \%$ of GPs in cycle 1 and $51.6 \%$ of GP in cycle $2(\mathrm{P}=0.0001)$.

Constipation, as measured by combining all patient data sources the Bowel Function Index, stool frequency and the Bristol Stool Scale, was very common amongst the patients prescribed strong opioids with 50.5\% (477/944) of patients across both audit cycles reporting symptoms of being constipated. The prevalence of OIC did not differ significantly between the two audit cycles (cycle $1,52.4 \%$ cycle 2 , 46.7\%). For patients audited in both cycles, OIC affecting quality of life or pain management occurred in $20.8 \%(196 / 944)$ of patients. The prevalence of OIC differed between the different methods of assessing constipation. It was lowest, when based only on stool frequency $(11.2 \%)$ and highest when assessed with the Bowel Function Index (43.1\%).

There was a marked difference in the presence of OIC as reported by patients and GPs. Half of patients reported symptoms of OIC, but GPs believed that only $22.8 \%$ of their patients were currently constipated. There was a trend for improved GP awareness of OIC in cycle 2, with a higher level of agreement between GP and patient reporting of current constipation, increasing from $61 \%$ in cycle 1 to $67 \%$ in cycle $2(\mathrm{P}=0.075)$.

To investigate any dose relationship for OIC, the oral morphine equivalent dose per day was calculated [14] and opioids were grouped as being low ( $\leq 40 \mathrm{mg} /$ day), medium (41-100 mg/day) or high dose (>100 mg/day). There was no dose relationship with respect to OIC as the prevalence of OIC was $52.9 \%$ amongst patients taking low doses compared to $51.5 \%$ for high doses $(\mathrm{P}=0.744)$. There was potentially a relationship between opioid dose and problematic OIC (impacting quality of life or pain management) (Figure 2).

The use of opioid antagonists and laxatives increased significantly from cycle 1 to cycle 2 ( $16.9 \%$ to $24.8 \%, \mathrm{P}=0.004$; and $42.9 \%$ to $50.0 \%$, $\mathrm{P}=0.041$ respectively). The majority of patients taking laxatives where using them regularly (every day or second day). The three most commonly used laxatives were osmotic laxatives (16.5\%), the combination of a stool softener plus a stimulant laxative $(7.7 \%)$ and bulk-forming agents (7.4\%). The laxative used was most commonly that recommended by their GP; $69.1 \%$ in cycle 1 increasing to $78.0 \%$ in cycle $2(\mathrm{P}=0.048) .47 .2 \%$ of patients were very or completely satisfied with their laxatives, whilst $12.8 \%$ patients were not satisfied or only a little satisfied.

\section{Discussion}

The GP assessment and management of OIC improved as result of participating in this clinical audit. The greatest improvements were observed for the co-prescribing a therapy to manage OIC (opioid antagonist or laxative) at the time of opioid initiation and for increasing the frequency of the assessment of OIC. Despite these improvements, there is need for increased focus on the proactive management of OIC as this occurred in less than half of patients audited in cycle 2, whilst best practice recommends that OIC is anticipated and proactively managed in all patients $[9,10,15]$.

In this Australian GP patient population with CNCP, OIC was very common occurring in $50.5 \%$ of patients. GPs underestimated the prevalence of OIC, only identifying $22.8 \%$ of patients as being currently constipated. Specific reasons for this underestimation cannot be directly elucidated from the audit; however constipation is a subjective experience [2] and if its assessment is limited to objective measures, such as stool frequency, then many patients with constipation may not be identified. Many patients may not discuss constipation with their GP or under-report their symptoms due to embarrassment or a lack of awareness that OIC may result in adverse clinical outcomes. Taking a history of constipation and regularly asking about OIC are essential steps to overcoming this clinical barrier [16]. Even though this audit focused on OIC associated with the use of strong opioids, GPs need to apply the same principles to patient's prescribed weak opioids in particular codeine due to its high propensity to cause constipation.

The audit used a combination of objective and subjective measures to evaluate constipation [2]. This included gathering information about the frequency of bowel movements, stool consistency and color, concomitant symptoms, diet and fluid intake and medication use, including laxatives [17]. Asking patients the questions from the validated Bowel Function Index [18] identified the presence of constipation in more patients than via stool frequency or the Bristol Stool Scale. Hence, GPs should consider routinely asking all patients 


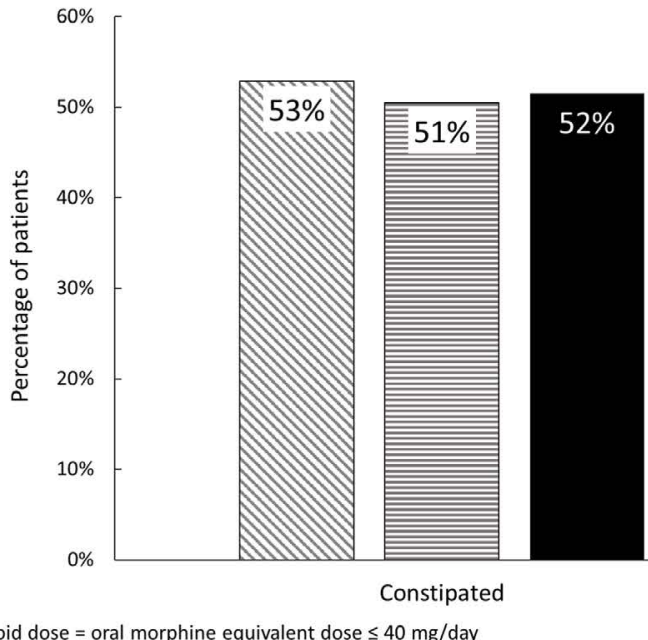

$\triangle$ Low opioid dose ( $n=448$ patients)

Medium opiod dose $(\mathrm{n}=216$ patients)

- High opioid dose ( $\mathrm{n}=194$ patients)

Medium opioid dose $=$ oral morphine equivalent dose $41-100 \mathrm{mg} /$ day

High opioid dose $=$ oral morphine equivalent dose $>100 \mathrm{mg} /$ day

Figure 2: Relationship between opioid dose and constipation

taking opioids about the ease or difficulty of defaecation, feelings of incomplete bowel evacuation and the patient's personal judgement of constipation.

One in five patients had OIC which was significantly affecting their quality of life and/or pain management. This is concerning, as these patients were receiving standard care yet many were suffering from a manageable side effect. This level of interference is lower than has been reported in other surveys [3].

Management options for OIC include life style measures, the use of laxatives, the co-administration of locally acting (enteric) opioid antagonists and opioid rotation [19]. All patients should be advised of life style measures (adequate fluids, fibre, exercise), however these measures alone are rarely effective to relieve OIC [10]. Laxatives have traditionally been used first line, but they often provide suboptimal relief, with $50 \%$ of patients failing to achieve a satisfactory response $[10,19,20]$. Reducing the opioid dose is not an effective strategy as the dose required to induce OIC is often less than that required for adequate analgesia [19]. In addition, as demonstrated in this audit, OIC was just as common in patients taking low or high opioid doses. Rotation to an opioid that is potentially less constipating is an appealing management strategy. However in this audit, there were no clear differences in the propensity of individual opioids to cause OIC, including transdermal opioids. The use of locally acting opioid antagonists, such as the combination of modified-release oxycodone/naloxone is an effective method of reducing the incidence and burden of OIC [21-23].

The main limitation of this research is in the nature of a clinical audit. It took two snapshots of clinical management and even though GP performance improved, it's unknown if this translated into improved patient outcomes. Similarly the longevity of the improved clinical practice is unknown.

\section{Conclusions}

OIC is common affecting 1 in 2 Australian patients prescribed strong opioids. GPs failed to recognize OIC in a third of their patients. GPs need to regularly ask all patients taking opioid analgesics about the presence of constipation. Questioning should include both objective measures, e.g. stool frequency, and subjective measures e.g. ease of defaecation, feelings of incomplete bowel movements and the patient's personal judgement of constipation.

OIC needs to be proactively managed from the time of opioid initiation and for the duration of opioid therapy. To paraphrase an old aphorism, 'the hand that writes the opioid, should simultaneously write the treatment for OIC'

\section{References}

1. Walsh TD (1990) Prevention of opioid side effects. J Pain Symptom Manage 5: 362-367.

2. Brock C, Olesen SS, Olesen AE, Frøkjaer JB, Andresen T, et al. (2012) Opioidinduced bowel dysfunction: pathophysiology and management. Drugs 72 : 1847-1865.

3. Bell TJ, Panchal SJ, Miaskowski C, Bolge SC, Milanova T, et al. (2009) The prevalence, severity, and impact of opioid-induced bowel dysfunction: results of a US and European Patient Survey (PROBE 1). Pain Med 10: 35-42.

4. Tuteja AK, Biskupiak J, Stoddard GJ, Lipman AG (2010) Opioid-induced bowe disorders and narcotic bowel syndrome in patients with chronic non-cancer pain. Neurogastroenterol Motil 22: 424-430, e96.

5. Kalso E, Edwards JE, Moore RA, McQuay HJ (2004) Opioids in chronic noncancer pain: systematic review of efficacy and safety. Pain 112: 372-380.

6. Allan L, Hays H, Jensen NH, de Waroux BL, Bolt M, et al. (2001) Randomised crossover trial of transdermal fentanyl and sustained release oral morphine for treating chronic non-cancer pain. BMJ 322: 1154-1158.

7. Moore RA, McQuay HJ (2005) Prevalence of opioid adverse events in chronic non-malignant pain: systematic review of randomised trials of oral opioids. Arthritis Res Ther 7: R1046-1051.

8. McNicol E, Horowicz-Mehler N, Fisk RA, Bennett K, Gialeli-Goudas M, et al. (2003) Management of opioid side effects in cancer-related and chronic noncancer pain: a systematic review. J Pain 4: 231-256.

9. Benyamin R, Trescot AM, Datta S, Buenaventura R, Adlaka R, et al. (2008) Opioid complications and side effects. Pain Physician 11: S105-120.

10. Pappagallo M (2001) Incidence, prevalence, and management of opioid bowe dysfunction. Am J Surg 182: 11S-18S.

11. Thorpe DM (2001) Management of opioid-induced constipation. Curr Pain Headache Rep 5: 237-240. 
Citation: Katelaris P, Krassas G (2016) The Clinical Challenge of Opioid-induced Constipation: Insights from the Opioid-induced Constipation Clinical Audit. J Gen Pract 4: 221. doi: 10.4172/2329-9126.1000221

Page 5 of 5

12. Swegle JM, Logemann C (2006) Management of common opioid-induced adverse effects. Am Fam Physician 74: 1347-1354.

13. Holliday S, Magin P, Dunbabin J, Oldmeadow C, Henry JM, et al. (2013) An evaluation of the prescription of opioids for chronic nonmalignant pain by Australian general practitioners. Pain Med 14: 62-74.

14. (2014) Faculty of Pain Medicine, Australian and New Zealand College of Anaethetists, Opioid dose equivalence.

15. Chou R, Fanciullo GJ, Fine PG, Adler JA, Ballantyne JC, et al. (2009) Clinical guidelines for the use of chronic opioid therapy in chronic noncancer pain. $J$ Pain 10: 113-130.

16. Sharma A, Jamal MM (2013) Opioid induced bowel disease: a twenty-first century physicians' dilemma. Considering pathophysiology and treatment strategies. Curr Gastroenterol Rep 15: 334.

17. Leppert W (2010) The role of opioid receptor antagonists in the treatment of opioid-induced constipation: a review. Adv Ther 27: 714-730.

18. Rentz AM, Yu R, Müller-Lissner S, Leyendecker P (2009) Validation of the Bowel Function Index to detect clinically meaningful changes in opioid-induced constipation. J Med Econ 12: 371-383.
19. Ketwaroo GA, Cheng V, Lembo A (2013) Opioid-induced bowel dysfunction. Curr Gastroenterol Rep 15: 344.

20. Kurz A, Sessler DI (2003) Opioid-induced bowel dysfunction: pathophysiology and potential new therapies. Drugs 63: 649-671.

21. Löwenstein O, Leyendecker P, Hopp M, Schutter U, Rogers PD, et al. (2009) Combined prolonged-release oxycodone and naloxone improves bowel function in patients receiving opioids for moderate-to-severe non-malignant chronic pain: a randomised controlled trial. Expert Opin Pharmacother 10: 531 . 543.

22. Löwenstein O, Leyendecker P, Lux EA, Blagden M, Simpson KH, et al. (2010) Efficacy and safety of combined prolonged-release oxycodone and naloxone in the management of moderate/severe chronic non-malignant pain: results of a prospectively designed pooled analysis of two randomised, double-blind clinical trials. BMC Clin Pharmacol 10: 12.

23. Simpson K, Leyendecker P, Hopp M, Müller-Lissner S, Löwenstein O, et al. (2008) Fixed-ratio combination oxycodone/naloxone compared with oxycodone alone for the relief of opioid-induced constipation in moderate-to-severe noncancer pain. Curr Med Res Opin 24: 3503-3512. 